\title{
A Note on Japanese Names
}

Japanese names appear in the text in Japanese order, with the family name preceding the personal name. To avoid confusion, I have followed this practice throughout, whether Japanese scholars have published in Japanese or in English. The bibliography follows American custom, with authors listed in alphabetical order by family name. 
